\title{
Can Physical Activity Reduce the Risk of Cognitive Decline in Apolipoprotein e4 Carriers? A Systematic Review
}

\author{
Jose Luis Perez-Lasierra ${ }^{1,2,3,+}+\mathbb{D}$, Jose Antonio Casajús $1,2,3,4,+\mathbb{D}$, José Antonio Casasnovas ${ }^{5,6} \mathbb{D}$, \\ Jose Miguel Arbones-Mainar ${ }^{4,5,7}$, Antonio Lobo ${ }^{5,8,9}\left(\mathbb{D}\right.$, Elena Lobo ${ }^{5,8,10}$ (D), Belén Moreno-Franco ${ }^{5,6,10}(\mathbb{D}$ \\ and Alejandro Gonzalez-Agüero $1,2,3,4, *,+$ (D)
}

\section{check for} updates

Citation: Perez-Lasierra, J.L.; Casajús, J.A.; Casasnovas, J.A.; Arbones-Mainar, J.M.; Lobo, A.; Lobo, E.; Moreno-Franco, B.;

Gonzalez-Agüero, A. Can Physical Activity Reduce the Risk of Cognitive Decline in Apolipoprotein e4 Carriers? A Systematic Review. Int. J. Environ. Res. Public Health 2021, 18 7238. https://doi.org/10.3390/ ijerph18147238

Academic Editors:

Fernando Sánchez-Santed, Maria

Teresa Colomina Fosch,

Caridad López Granero and

Ingrid Reverte

Received: 21 April 2021

Accepted: 1 July 2021

Published: 6 July 2021

Publisher's Note: MDPI stays neutral with regard to jurisdictional claims in published maps and institutional affiliations.

Copyright: (c) 2021 by the authors. Licensee MDPI, Basel, Switzerland. This article is an open access article distributed under the terms and conditions of the Creative Commons Attribution (CC BY) license (https:// creativecommons.org/licenses/by/ $4.0 /)$.
1 Department of Physiatry and Nursing, Universidad de Zaragoza, 50009 Zaragoza, Spain; jlperez@unizar.es (J.L.P.-L.); joseant@unizar.es (J.A.C.)

2 GENUD (Growth, Exercise, Nutrition and Development) Research Group, 50009 Zaragoza, Spain

3 Instituto Agroalimentario de Aragón (IA2), 50013 Zaragoza, Spain

4 CIBEROBN Instituto de Salud Carlos III, 28029 Madrid, Spain; adipofat@gmail.com

5 Instituto de Investigación Sanitaria Aragón, Hospital Universitario Miguel Servet, 50009 Zaragoza, Spain; jacasas@unizar.es (J.A.C.); alobosat@gmail.com (A.L.); elobo@unizar.es (E.L.); mbmoreno@unizar.es (B.M.-F.) 6 CIBERCV Instituto de Salud Carlos III, 28029 Madrid, Spain

Adipocyte and Fat Biology Laboratory (AdipoFat), 50009 Zaragoza, Spain

CIBERSAM Instituto de Salud Carlos III, 28029 Madrid, Spain

9 Department of Medicine and Psychiatry, Universidad de Zaragoza, 50009 Zaragoza, Spain

10 Department of Microbiology, Pediatrics, Radiology and Public Health, Universidad de Zaragoza, 50009 Zaragoza, Spain

* Correspondence: alexgonz@unizar.es; Tel.: +34-976761000

+ EXERNET Red de Investigación en Ejercicio Físico y Salud, Spain.

Abstract: Physical activity (PA) reduces the risk of cognitive decline (CD) in the general population. However, little is known about whether the presence of the apolipoprotein E epsilon 4 allele (APOE e4) could modify this beneficial effect. The aim of this systematic review was to analyze and synthetize the scientific evidence related to PA levels and CD risk in cognitively healthy APOE e4 carriers. Four electronic databases were analyzed. Only original articles with longitudinal study design were selected to analyze the relationship between PA and CD in APOE e4 carriers. Five studies were included in the systematic review. All studies except one stated that PA is a protective factor against $\mathrm{CD}$ in $A P O E$ e 4 carriers. Moreover, partial support was found for the hypothesis that a greater amount and intensity of PA are more beneficial in CD prevention. The results support the idea that PA is a protective factor against CD in APOE e4 carriers. Nevertheless, it would be necessary to carry out further studies that would allow these findings to be contrasted.

Keywords: exercise; APOE e4; Cognitive Dysfunction; mild cognitive impairment

\section{Introduction}

Cognitive decline (CD) in older adults is an ever-growing problem because the number of older adults is increasing [1] and because longer life increases the likelihood of loss of memory and decline in the performance of cognitive tasks [2,3]. Cognitive performance is considered an important aspect of healthy aging [4]. There is an increasing interest in this subject, which is now a priority in public health [5], and the European Innovation Partnership on Active and Health Aging [6], has also recognized its relevance. Therefore, the prevention of CD should be an area of increasing interest for research in public health.

Mild cognitive impairment (MCI) is one construct of CD that has received considerable attention in the literature, mainly because it has been widely sustained that MCI progresses to dementia, Alzheimer's disease in particular, in a high proportion of individuals [7], and also because of its high prevalence, even in the community. Several studies have suggested the idea that CD, which includes MCI, may be considered a health index because both 
have been associated with an increased mortality rate in the general population [8-10]. Moreover, it has been shown that $\mathrm{CD}$ is associated with disability in the general population, even after controlling for dementia [11]. Consequently, the prevention of $C D$ should be a subject of particular attention.

A multitude of risk factors of different typology have been associated with CD [2,12,13]; age is the most important one [3], although cardiovascular diseases are also risk factors $[2,12,14]$. Other characteristics such as low educational level [12], depression and anxiety $[10,15,16]$, vitamin B12 or D deficiency [12] as well as certain lifestyles that include smoking and physical inactivity [14] may also play a role in CD.

Among the previously documented risk factors of $C D$, a major genetic risk factor is the presence of the apolipoprotein E epsilon 4 allele (APOE e4) [2,17]. The human apolipoprotein $\mathrm{E}$ gene is polymorphic, presenting three alleles: epsilon 3 (e3), epsilon 2 (e2) and epsilon 4 (e4). The $e 3$ variant is the most frequent allele $(\sim 77 \%$ in the general population), while $e 2$ and $e 4$ are less common, with occurrence rates of $7.8 \%$ and $15.1 \%$, respectively [18]. APOE e4 presence is associated with several risk factors and diseases [19-22], including faster CD [2,23]. Homozygotes have the highest risk, followed by heterozygotes, implying that the effect of $A P O E$ e 4 on CD is dose-dependent $[17,24]$. The underlying mechanisms by which APOE e4 is associated with early CD suggest that $A P O E$ e4 is deficient in beta-amyloid clearance and accelerates beta-amyloid deposition to form amyloid plaques in the brain [20]. The toxic amyloid plaques injure synapses and ultimately cause neurodegeneration and CD [25].

On the other hand, protective factors of CD have also been reported [12,14,26], including intellectual activity and lifestyles aspects such as social interaction and physical activity (PA) $[27,28]$. PA may act on several risk factors related to $C D$, and a relationship between PA and neurogenesis, plasticity and higher white matter volume has been shown [29-31]. Several studies demonstrate how cardiovascular diseases and their risk factors are associated with $\mathrm{CD}$ and lower performance in multiple cognitive domains [32,33]. However, PA is beneficial in the prevention of cardiovascular diseases and their risk factors [34-36], as well as other known risk factors for $\mathrm{CD}$ such as depression or anxiety $[35,37]$. Therefore, PA may indirectly prevent $\mathrm{CD}$ by counteracting the $\mathrm{CD}$ risk factors. It has also been reported that PA is beneficial for the prevention of MCI and CD in the general population [12,29,31,38,39]. Recent meta-analyses also suggest that PA reduces the risk of suffering CD [27,40]; however, none of the studies reviewed were differentiated according to the genotyping of the participants based on the presence of APOE e4. This is a source of concern, since perhaps the effect of PA in subjects with high genetic risk of $\mathrm{CD}, A P O E$ e4 carriers, is different from the effect that PA has in subjects without genetic risk, APOE e4 non-carriers. In order to create useful strategies for the prevention of severe neurodegenerative diseases, it is crucial to investigate the role that PA may have against neurodegeneration in these subjects with increased genetic risk.

Some studies have analyzed to what extent the amount and intensity of PA influence its potential beneficial effect for the prevention of $C D$ in the general population. Several studies have suggested that higher levels of PA (exercise engaged in $\geq 3$ days/week at intensity greater than walking) or vigorous intensity PA is more beneficial [41-44], but discrepant results have also been reported [40], and the question is not settled.

On the basis of the previous reports, the main aim of this systematic review was to analyze and synthetize the scientific evidence related to PA levels and CD risk in cognitively healthy APOE e4 subjects (without previous $\mathrm{CD}$ ) and to review the literature that investigates whether the effect of PA in these subjects is directly associated with amount and intensity.

\section{Materials and Methods}

This work used the model of preferred reporting items for a systematic review and meta-analysis (PRISMA) [45] to ensure accuracy and comprehensiveness. A review protocol was written prior to reviewing the literature. 
As all analyses were based on publicly available summary statistics, no ethical approval from an institutional review board or informed patient consent was required.

Four electronic databases were analyzed systematically using different keywords and Boolean operators. The databases analyzed were PubMed, SportDiscus, Cochrane Library and Web of Science (WOS). The strategy included searching by index terms (MeSH) and by free text. The search by index terms used in PubMed was: "Exercise" [Mesh] AND "Cognitive Dysfunction" [Mesh] AND "Apolipoprotein E4" [Mesh]. The search by free text used in PubMed, SportDiscus, Cochrane Library and WOS was: "Physical Activity" OR "Exercise") AND ("Memory impairment" OR "Age-associated memory impairment" OR "Late-life forgetfulness" OR "age-related cognitive decline" OR "Mild Cognitive Impairment" OR "Cognitive Decline" OR “Cognitive Dysfunction") AND ("Apolipoprotein E4" OR "Apoe4" OR "Apo E4" OR "Apoe 4" OR "Apoe epsilon 4" OR "Apolipoprotein E-4" OR “Apo E-4" OR “Apo E 4" OR “Apolipoprotein E epsilon4").

For the present systematic review, only original articles published up to 13 April 2021 were analyzed, excluding all types of symposium reports, letters to the editor, conference abstracts, books, opinions of experts and reviews of any kind. The inclusion criteria used were that the studies covered the theme of the present review (relationship between the risk of $\mathrm{CD}$ (which includes cases of MCI), and PA in participants genotyped with $e 4$ allele), were longitudinal studies, used humans as participants, were published in English and that the sample used was composed of people without brain injuries or diagnosed mental disorders at baseline.

Studies were excluded if they did not provide information on how the cognitive function or the PA was evaluated, if the PA was evaluated jointly with the rest of the activities carried out in the free time (including sedentary leisure activities) or if they were related to dementia and not specifically to $C D$. Papers that did not specifically assess the impact of PA on CD in APOE $e 4$ and those related to animal experimentation were all excluded.

Applying these criteria and using the search strategy described, the process of article selection is illustrated in Figure 1.

Two reviewers (J.L.P.-L. and A.G.A.) carried out the selection process independently based on the criteria previously established. Any discrepancies were resolved by consensus, and in some cases, a third reviewer (J.A.C.) was consulted to resolve disagreements. Starting from the initial search after applying the search criteria and strategies, the articles were first selected based on the title and abstract, identifying key and thematic keywords related or unrelated to our aim, then those duplicated articles were eliminated, and the last screening was performed after a complete and exhaustive reading of the full text, selecting the papers to be included in our systematic review. Finally, after the article selection process, the bibliography of the articles was reviewed in order to identify articles that could meet our inclusion criteria.

Data from the studies, such as the longitudinal follow-up time, the population sample used and its characteristics (average age of the participants in the baseline, sex and genotype), the cognitive function and PA evaluation method, the covariates (confounders) used in the studies, the study design and the main results, were extracted based on a second reading of the full text of the articles included in the systematic review. Moreover, the results of the included studies were grouped according to whether they compared the risk for $\mathrm{CD}$ among different PA levels only in $e 4$ carriers or in $e 4$ carriers vs. non-carriers. Participants of the studies were considered APOE e4 carriers if they carried at least one e4 allele. 


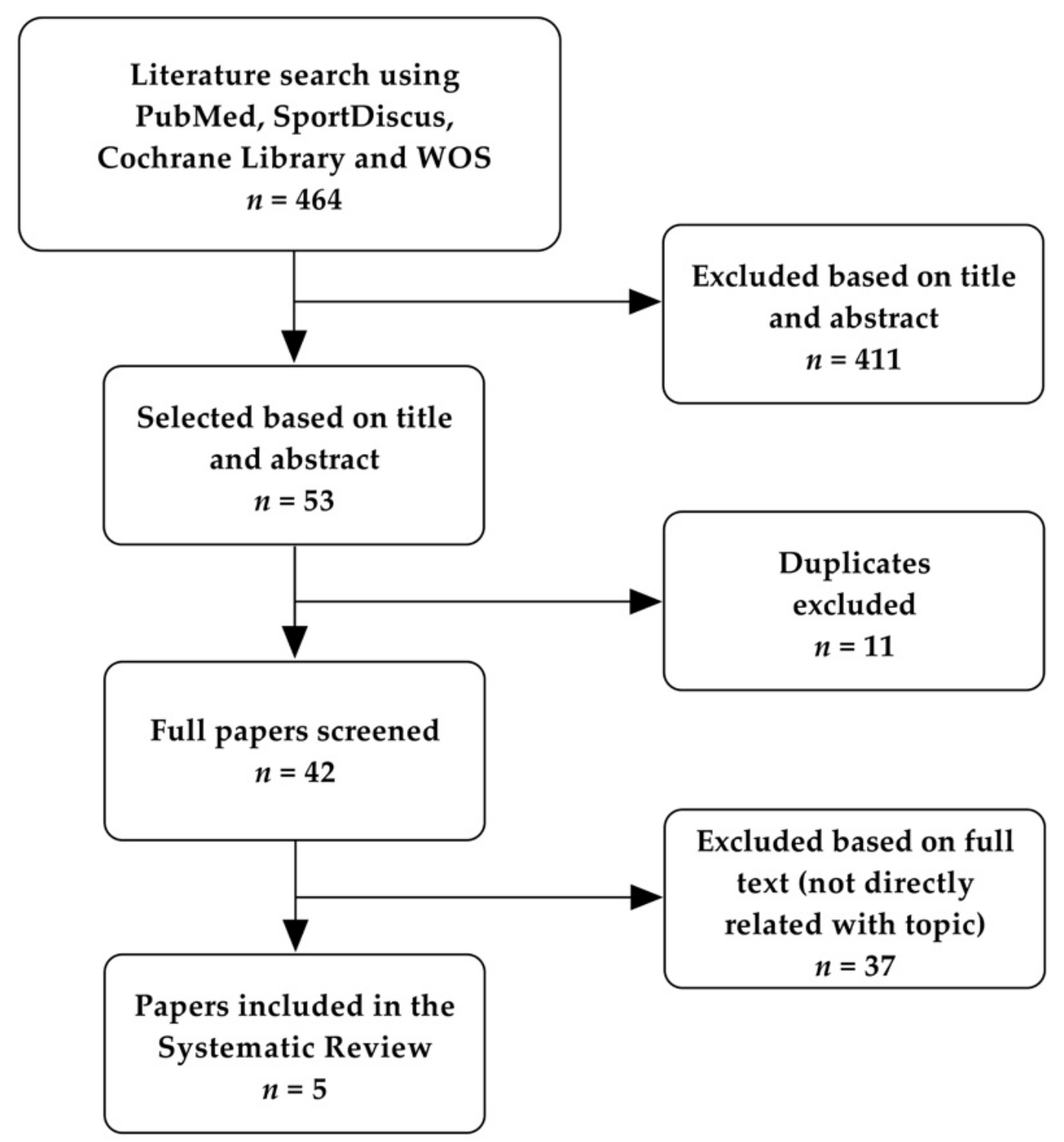

Figure 1. Flow diagram of search strategy in databases (PubMed, SportDiscus, Cochrane Library and Web of Science). Note: WOS-Web of Science.

Quality assessment was performed using the Newcastle-Ottawa Scale (NOS) for cohort studies [46] and the PEDro scale for randomized controlled trials [47]. The NOS for cohort studies uses a "star" rating system to judge quality based on three aspects of the study: (a) selection of participants, (b) comparability of study groups and (c) outcome of interest. The maximum number of stars a study may receive in each of these three categories is 4, 2 and 3, respectively. The highest rating a study can receive is 9 stars. A detailed description of the NOS criteria for assessing quality can be found in the Supplementary Materials.

The PEDro scale consists of 11 criteria that assess the methodological quality of the experimental studies based on three aspects: internal validity (criteria 2-9), interpretability (criteria 10-11) and external validity or applicability (criterion 1).

In the NOS, we assigned scores of $0-3,4-6$ and 7-9 to indicate low, moderate and high-quality studies, respectively, and, in the PEDro scale, we assigned scores of 0-4, 5-8, and 9-11 to indicate low, moderate and high-quality studies, respectively. 


\section{Results}

\subsection{Study Selection}

As shown in Figure 1, the initial electronic database search yielded 464 hits in total. A total of 411 articles were excluded following screening titles and abstracts, 11 articles were removed for duplicates and 37 articles were excluded following screening of full texts. A total of five articles from the electronic search met the inclusion criteria, and, after the manual search, no articles were added.

\subsection{Description of Included Studies}

There are several differences in the design and the methodology of the different included studies. Four of them have a prospective cohort design [48-51], and the other is a randomized controlled trial [52]. Between-study differences were observed in the methods to assess the exposure and outcome. Moreover, the range of follow-up time among the studies is quite broad, from 18 months [51] to 10 years [52].

Several studies were carried out on Americans [48,50,52], one on Mexican-Americans [50] and another on American populations of Indian, African, Asian and Hispanic descent [52]. In the latter study, it should be noted that all the participants in the baseline had type II diabetes and overweight or obesity [52]. Another study was conducted on Caucasians [51], and the last one was carried out on Asians [49].

Regarding all the participants of the studies that compared the risk for $\mathrm{CD}$ among different PA levels in $e 4$ carriers vs. non-carriers (Table 1), a total of 7148 participants were included, and 1425 of them were APOE e4 carriers, 5043 were non-carriers and the remaining 680 were reported as having a missing or unknown genotype (nine study participants from one article [48], and 671 study participants from another [52]). Moreover, the population sample of the studies ranged from 78 participants [51] to 3802 participants [52].

Taking into account all the participants of the studies that compared the risk for $\mathrm{CD}$ among different PA levels in e4 carriers (Table 2), a total of 792 participants were included, but the samples of the studies also varied between 26 participants [51] and 474 participants [48].

\subsection{Assessment of Main Variables}

To assess PA, different types of questionnaires were used. Two of the studies used questionnaires created ad hoc that were not validated [49,50], while the others used validated questionnaires such as the Minnesota Leisure Time Physical Activity Questionnaire and the 1985 National Health Interview Survey [48], Paffenbarger Physical Activity Questionnaire [52] or the Stanford Brief Activity Survey [51].

To assess cognitive function, all the studies used specific tests or questionnaires, and although there is considerable diversity, in all the studies except one [48], the Mini-Mental State Examination (MMSE) [49,51], or its modified version, the Modified Mini-Mental State Examination (3MSE) [50,52], were used, both of which are employed worldwide [53].

Tables 1 and 2 show the questionnaire used in each of the studies to assess PA, cognitive function and the criteria for establishing $\mathrm{CD}$ or $\mathrm{MCI}$ based on the cognitive assessment tests used throughout the follow-up. Three of the included studies used MMSE or 3MSE cutoff points to define CD [49,50,52], another used Petersen's criteria for MCI [48] and the remaining study used cutoff points in at least one of the principal outcome indices (DRS-2 and RAVLT) [51]. Tables 1 and 2 display the different criteria used in each study to classify the participants among the different PA levels.

\subsection{Risk of Bias Assessment}

There was a heterogeneous quality in the methodology of the included studies, since two had high quality $[48,50]$, and the others had moderate quality $[49,51,52]$ (Table 3). 
Table 1. Summary of the reviewed articles that compare the risk for CD among different PA levels in APOE e4 carriers vs. non-carriers.

\begin{tabular}{|c|c|c|c|c|c|c|c|c|c|c|c|c|c|}
\hline \multirow[b]{2}{*}{$\begin{array}{c}\text { Study, } \\
\text { (Study Design) }\end{array}$} & \multirow[b]{2}{*}{$\begin{array}{c}\text { Follow-Up, } \\
\text { y, Mean, (Range) }\end{array}$} & \multirow[b]{2}{*}{$\begin{array}{l}\text { Method to } \\
\text { Assess PA }\end{array}$} & \multirow[b]{2}{*}{$\begin{array}{l}\text { Method to } \\
\text { Assess Cognitive } \\
\text { Function }\end{array}$} & \multirow[b]{2}{*}{$\begin{array}{l}\text { Adjudication of } \\
\text { CD or MCI }\end{array}$} & \multirow[b]{2}{*}{ Confounders } & \multirow[b]{2}{*}{$\begin{array}{c}\text { Study } \\
\text { Subgroups }\end{array}$} & & \multirow[b]{2}{*}{ Main Results } & \multicolumn{5}{|c|}{ Sample } \\
\hline & & & & & & & & & $n$ & $\begin{array}{c}\text { Female Sex, } \\
n(\%)\end{array}$ & $\begin{array}{l}\text { Age, } y, \\
\text { mean } \\
\text { (SD), } \\
\text { and/or } \\
\text { Range } \\
\end{array}$ & $\begin{array}{c}A P O E \text { e } 4, \\
n, \\
(e 4 e+, n)\end{array}$ & $\begin{array}{c}\text { No } \\
\text { APOE } e^{4},\end{array}$ \\
\hline $\begin{array}{c}\text { Espeland, } \mathrm{M} \text {. } \\
\text { et al. } \\
\text { (intervention) }\end{array}$ & $\begin{array}{c}9.8 \\
(8.4-11.1)\end{array}$ & $\begin{array}{l}\quad \text { - Paffebarger Physical } \\
\text { Activity } \\
\text { Questionnaire }\end{array}$ & $\begin{array}{l}-3 \mathrm{MSE} \\
-\mathrm{FAQ}\end{array}$ & $\begin{array}{l}\text { 3MSE test score fell } \\
\text { below prespecified } \\
\text { age-and } \\
\text { education-specific } \\
\text { cutoff points }\end{array}$ & - Age & $\begin{array}{l}\text { - Groups by: } \\
\text { - Genotype } \\
\text { - Randomization: } \\
\text { - Intervention: }>175 \text { min } \\
\text { PA/week brisk } \\
\text { walking } \\
\text { - Control: PA, diet and } \\
\text { social support }\end{array}$ & \multicolumn{2}{|r|}{$\begin{array}{l}\text { - Non-carriers control group OR }=1.00 \text { (ref. } \\
\text { group) } \\
\text { - Carriers control group } \mathrm{OR}=\mathrm{NI} \\
\text { - Carriers intervention group } \mathrm{OR}=0.84 \\
(95 \% \mathrm{Cl}: 0.52,1.36, p>0.05 \text { ) }\end{array}$} & 3802 & $\begin{array}{l}2323 \\
(61)\end{array}$ & $45-76$ & $\begin{array}{l}724 \\
(57)\end{array}$ & 2407 \\
\hline \multirow{6}{*}{$\begin{array}{c}\text { Krell, J. et al. } \\
\text { (observational) }\end{array}$} & \multirow{6}{*}{$\begin{array}{c}3.2 \\
(1.9-4.7)\end{array}$} & \multirow{6}{*}{$\begin{array}{l}\text { - Minnesota leisure time } \\
\text { physical activity } \\
\text { questionnaire } \\
\text { - } 1985 \text { National Health } \\
\text { Interview Survey }\end{array}$} & \multirow{6}{*}{$\begin{array}{l}\text { - Neurological } \\
\text { evaluation } \\
\text { - STMS } \\
\text { - WAIS-R } \\
\text { - WMS-R }\end{array}$} & \multirow{6}{*}{$\begin{array}{l}\text { Petersen criteria } \\
\text { for MCI } 2004\end{array}$} & & \multirow{6}{*}{$\begin{array}{l}\text { - Groups by: } \\
\text { - Genotype } \\
\text { - Intensity of PA: } \\
\text { - LPA } \\
\text { - MPA } \\
\text { - VPA } \\
\text { - Moment in life when PA was } \\
\text { performed: } \\
\text { - Midlife } \\
\text { - Late life }\end{array}$} & $\begin{array}{l}\text { Midlife } \\
\text { and } \\
\text { LPA }\end{array}$ & 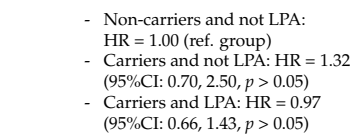 & \multirow{6}{*}{1830} & \multirow{6}{*}{$\begin{array}{l}919 \\
(50.22)\end{array}$} & \multirow{6}{*}{$\begin{array}{c}78 \\
74-83\end{array}$} & \multirow{6}{*}{$\begin{array}{l}474 \\
(\mathrm{NI})\end{array}$} & \multirow{6}{*}{1347} \\
\hline & & & & & & & $\begin{array}{l}\text { Midlife } \\
\text { and } \\
\text { MPA }\end{array}$ & 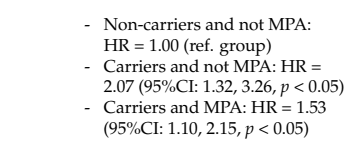 & & & & & \\
\hline & & & & & $\begin{array}{l}- \text { Age } \\
- \text { Sex } \\
- \text { Educational } \\
\text { level }\end{array}$ & & $\begin{array}{l}\text { Midlife } \\
\text { and } \\
\text { VPA }\end{array}$ & 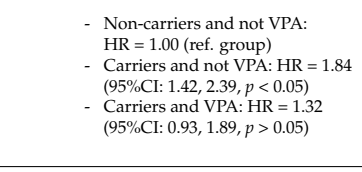 & & & & & \\
\hline & & & & & $\begin{array}{l}\text { Comorbidities } \\
\text { Depression }\end{array}$ & & $\begin{array}{l}\text { Late } \\
\text { life } \\
\text { and } \\
\text { LPA }\end{array}$ & 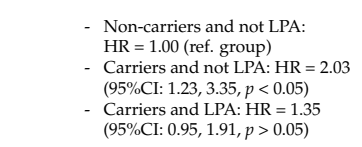 & & & & & \\
\hline & & & & & & & $\begin{array}{l}\text { Late } \\
\text { life } \\
\text { and } \\
\text { MPA }\end{array}$ & 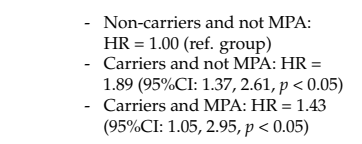 & & & & & \\
\hline & & & & & & & $\begin{array}{l}\text { Late } \\
\text { life } \\
\text { and } \\
\text { VPA }\end{array}$ & 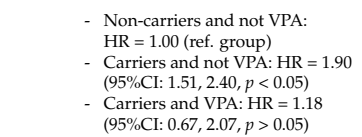 & & & & & \\
\hline
\end{tabular}


Table 1. Cont.

\begin{tabular}{|c|c|c|c|c|c|c|c|c|c|c|c|c|}
\hline \multirow[b]{2}{*}{$\begin{array}{l}\text { Study, } \\
\text { (Study Design) }\end{array}$} & \multirow[b]{2}{*}{$\begin{array}{c}\text { Follow-Up, } \\
\mathrm{y} \text {, Mean, (Range) }\end{array}$} & \multirow[b]{2}{*}{$\begin{array}{l}\text { Method to } \\
\text { Assess PA }\end{array}$} & \multirow[b]{2}{*}{$\begin{array}{l}\text { Method to } \\
\text { Assess Coggitive } \\
\text { Function }\end{array}$} & \multirow[b]{2}{*}{$\begin{array}{l}\text { Adjudication of } \\
\mathrm{CD} \text { or MCI }\end{array}$} & \multirow[b]{2}{*}{ Confounders } & \multirow[b]{2}{*}{$\begin{array}{l}\text { Study } \\
\text { Subgroups }\end{array}$} & \multirow[b]{2}{*}{ Main Results } & \multicolumn{5}{|c|}{ Sample } \\
\hline & & & & & & & & $n$ & $\begin{array}{l}\text { Female Sex, } \\
n(\%)\end{array}$ & $\begin{array}{l}\text { Age, } y, \\
\text { mean } \\
\text { (SD)/, } \\
\text { and/or } \\
\text { Range } \\
\end{array}$ & $\begin{array}{c}\text { APOE eq, } \\
n, \\
(e 4 e q, n)\end{array}$ & $\begin{array}{c}\text { No } \\
A P O E \text { eq, } \\
n\end{array}$ \\
\hline $\begin{array}{c}\text { Shih, I. et al. } \\
\text { (observational) }\end{array}$ & 6.5 & $\begin{array}{l}\text { - MET-h/week of } 18 \\
\text { common activities for } \\
\text { older adults (based on the } \\
\text { Compendium of Physical } \\
\text { activities) (not validated) }\end{array}$ & $\begin{array}{l}- \text { 3MSE } \\
- \text { SEVLT } \\
\text { (Delayed word } \\
\text { recall) } \\
- \text { SENAS }\end{array}$ & $\begin{array}{l}\text { Score 3MSE or } \\
\text { SEVLT fell less } \\
\text { than the 20th per- } \\
\text { centile/decreased } \\
\geq 8 \text { in } 3 \text { MSE or } 33 \\
\text { points in SEVLT } \\
\text { and scores less } \\
\text { than 20th } \\
\text { percentile at } \\
\text { follow-up }\end{array}$ & $\begin{array}{l}\text { - Age } \\
\text { - Sex } \\
\text { - Educational } \\
\text { level } \\
\text { - Diabetes } \\
\text { - Smoking } \\
\text { - History } \\
\text { of stroke } \\
\text { - Hours } \\
\text { stand- } \\
\text { ing/walking } \\
\text { at work }\end{array}$ & $\begin{array}{l}\text { Groups by: } \\
\text { - Genotype } \\
\text { - Level of PA: } \\
\text { - Low PA: } \\
\text { <35MT-h/week } \\
\text { - High PA: } \\
\text { >35MET-h/week }\end{array}$ & 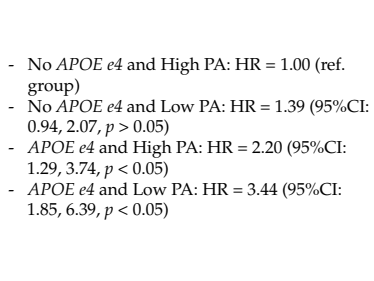 & 1438 & $\begin{array}{l}840 \\
(58)\end{array}$ & $69.7(6.2)$ & $\begin{array}{l}2011 \\
(11)\end{array}$ & 1237 \\
\hline $\begin{array}{c}\text { Woodard, J.L. } \\
\text { et al. } \\
\text { (observational) }\end{array}$ & 1.5 & $\begin{array}{l}\text { - Stanford Brief Activity } \\
\text { Survey }\end{array}$ & $\begin{array}{l}\text { - MMSE } \\
\text { - GDS } \\
\text { GDRS-2 } \\
\text { - RAVLT }\end{array}$ & $\begin{array}{l}\geq 1 \mathrm{SD} \text { reduction } \\
\text { on at least one of } \\
\text { the prinipal } \\
\text { outcomes indices } \\
\text { (DDSS-2, RAVITT } \\
\text { Sum of trials } 15 \text {, } \\
\text { RAVIT delayed } \\
\text { word recall) }\end{array}$ & NI & 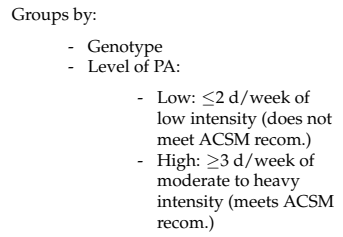 & 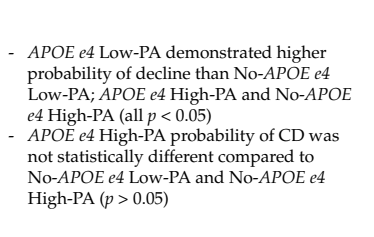 & 78 & $\begin{array}{l}57 \\
(73)\end{array}$ & $72.6(5.0)$ & $\begin{array}{l}26 \\
\text { (1) }\end{array}$ & 52 \\
\hline
\end{tabular}

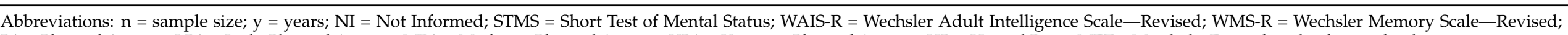

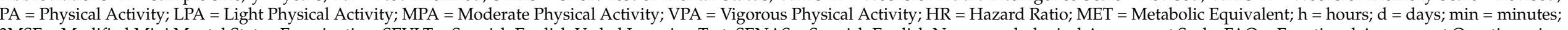

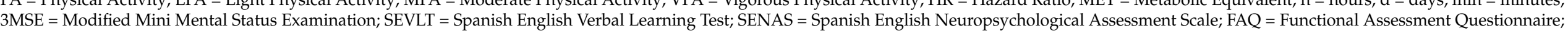

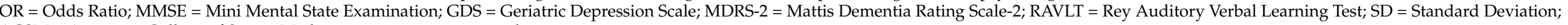
ACSM = American College of Sports Medicine; recom. = recommendations. 
Table 2. Summary of the reviewed articles that compare the risk for CD among different PA levels in APOE e4 carriers.

\begin{tabular}{|c|c|c|c|c|c|c|c|c|c|c|c|}
\hline \multirow{2}{*}{$\begin{array}{l}\text { Study, } \\
\text { (Study Design) }\end{array}$} & \multirow{2}{*}{$\begin{array}{c}\text { Follow-Up, } \\
\text { y, Mean, } \\
\text { (Range) }\end{array}$} & \multirow[b]{2}{*}{$\begin{array}{l}\text { Method to } \\
\text { Assess PA }\end{array}$} & \multirow[b]{2}{*}{$\begin{array}{l}\text { Method to Assess Cognitive } \\
\text { Function }\end{array}$} & \multirow[b]{2}{*}{$\begin{array}{l}\text { Adjudication of } \\
\text { CD or MCI }\end{array}$} & \multirow[b]{2}{*}{ Confounders } & \multirow[b]{2}{*}{$\begin{array}{l}\text { Study } \\
\text { Subgroups }\end{array}$} & \multirow{2}{*}{\multicolumn{2}{|c|}{ Main Results }} & \multicolumn{3}{|c|}{ Sample } \\
\hline & & & & & & & & & $\begin{array}{c}n \\
c^{\prime} \\
\left(e 4 e 4^{\prime}, n\right)\end{array}$ & $\begin{array}{c}\text { Female } \\
\text { Sex, } n(\%)\end{array}$ & $\begin{array}{l}\text { Age, } y, \text { Mean } \\
\text { (SD), and/or } \\
\text { Range }\end{array}$ \\
\hline $\begin{array}{l}\text { Krell, J. et al. } \\
\text { (observational) }\end{array}$ & $\begin{array}{l}3.2 \\
(1.9-4.7)\end{array}$ & $\begin{array}{l}\text { - Minnesota leisure } \\
\text { time physical } \\
\text { activity } \\
\text { questionnaire } \\
\text { - } 1985 \text { National } \\
\text { Health Interview } \\
\text { Survey }\end{array}$ & $\begin{array}{l}\text { - Neurological } \\
\text { evaluation } \\
\text { - STMS } \\
\text { - WAIS-R } \\
\text { - WMS-R }\end{array}$ & $\begin{array}{l}\text { Petersen criteria } \\
\text { for MCI } 2004\end{array}$ & $\begin{array}{l}\text { - Age } \\
\text { - Sex } \\
\text { - Educational } \\
\text { level } \\
\text { - Comorbidities } \\
\text { - Depression }\end{array}$ & $\begin{array}{l}\text { Groups by: } \\
\text { - Intensity of PA: } \\
\text { - LPA } \\
\quad \text { MPA } \\
\text { - VPA } \\
\text { - Moment in life when } \\
\text { PA was performed: } \\
\text { - Never } \\
\text { - In midiliee } \\
\text { - In late life } \\
\text { - Always }\end{array}$ & $\begin{array}{l}\text { MPA } \\
\text { VPA }\end{array}$ & 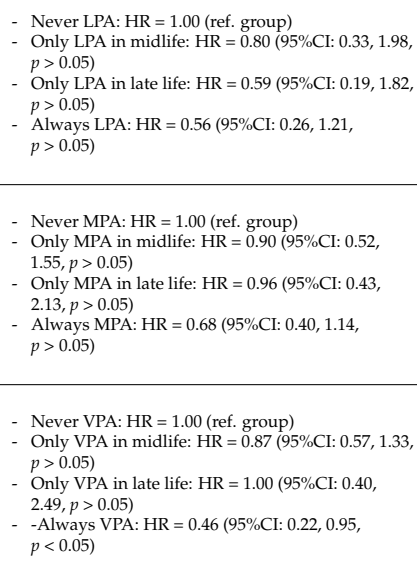 & $\begin{array}{l}474 \\
(\mathrm{NI})\end{array}$ & NI & 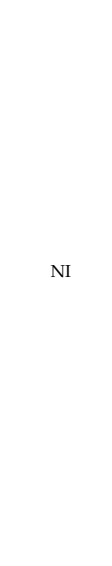 \\
\hline $\begin{array}{l}\text { Woodard, J.L.e et al. } \\
\text { (observational) }\end{array}$ & 1.5 & $\begin{array}{l}\text { - Stanford Brief } \\
\text { Activity Survey }\end{array}$ & $\begin{array}{l}- \text { MMSE } \\
- \text { GDS } \\
- \text { MDRS-2 } \\
- \text { RAVLT }\end{array}$ & $\begin{array}{l}\geq 1 \text { SD reduction on at } \\
\text { least one of the } \\
\text { principal outcomes } \\
\text { indices (DRS-2, , RAVLT } \\
\text { Sum of trials } 1-5, \\
\text { RAVLT delayed word } \\
\text { recall) }\end{array}$ & $\mathrm{NI}$ & 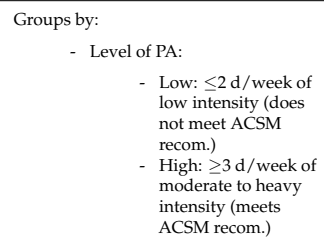 & & $\begin{array}{l}\text { Low-PA group demonstrated higher probability } \\
\text { of decline than High-PA group }(p<0.05)\end{array}$ & $\begin{array}{l}26 \\
\text { (1) }\end{array}$ & $\mathrm{NI}$ & $\mathrm{NI}$ \\
\hline
\end{tabular}

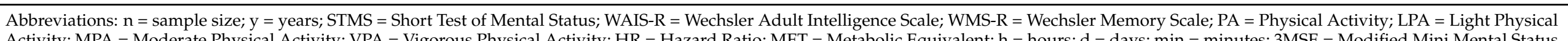

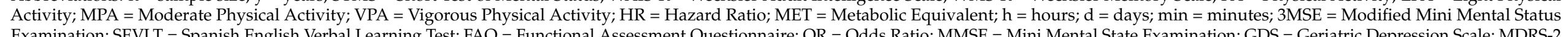

= Mattis Dementia Rating Scale-2; RAVLT = Rey Auditory Verbal Learning Test; ACSM = American College of Sports Medicine; recom. = recommendations; NI = Not Informed. 
Table 3. Newcastle-Ottawa and PEDro Quality Assessment of the studies included in the systematic review.

\begin{tabular}{|c|c|c|c|c|c|c|c|c|c|c|c|c|}
\hline \multirow{3}{*}{ Study } & \multicolumn{12}{|c|}{ Quality Assessment of Cohort Studies with NOS } \\
\hline & \multicolumn{4}{|c|}{ Selection } & \multicolumn{5}{|c|}{ Comparability Outcome } & \multirow{2}{*}{\multicolumn{3}{|c|}{ NOS QS }} \\
\hline & 1 & 2 & 3 & 4 & 5 & 6 & 7 & 8 & 9 & & & \\
\hline Krell, J. et al. & * & * & & * & * & * & * & & * & & 7 & \\
\hline Niti, M. et al. & * & * & & * & * & * & * & & & & 6 & \\
\hline Shih, I. et al. & * & * & & * & * & * & * & * & * & & 8 & \\
\hline Woodard, J.L. et al. & * & * & & * & & & * & & $*$ & & 5 & \\
\hline \multirow[b]{2}{*}{ Study } & \multicolumn{12}{|c|}{ Quality Assessment of RCT Studies with PEDro Scale } \\
\hline & 1 & 2 & 3 & 4 & 5 & 6 & 7 & 8 & 9 & 10 & 11 & $\begin{array}{c}\text { PEDro } \\
\text { QS }\end{array}$ \\
\hline Espeland, M. et al. & * & * & * & * & & & $*$ & & & $*$ & $*$ & 7 \\
\hline
\end{tabular}

\subsection{Risk of CD among Different PA Levels}

Two tables summarize the results of the included studies. Table 1 compares the studies that analyze the risk in $A P O E$ e4 carriers vs. non-carriers in relation to PA levels, and Table 2 compares the studies that analyze the risk between carriers with different PA levels.

Regarding the results provided in Table 1, Shih et al. stated that those carrying APOE $e 4$ who accomplished more than 35 MET-hours/week had a 2.20-fold increased hazard ratio (HR) $(95 \% \mathrm{CI}$ : 1.29, 3.74, $p<0.05)$, and those carrying APOE $e 4$ who performed less than 35 MET-hours/week had a 3.44-fold increased HR $(95 \% \mathrm{CI}: 1.85,6.39, p<0.05)$ of developing cognitive impairment compared to persons who were not APOE e4 carriers and who performed more than 35 MET-hours/week [50]. Krell-Roesch et al. stratified their results based on the intensity of PA (light, moderate and vigorous) and also based on the moment in life when PA was performed (midlife or late life). Based on this stratification, no differences in MCI risk were found for light and vigorous PA performed in midlife or late life between $A P O E$ e 4 carriers and non-carriers (Table 1). Nevertheless, when compared to $A P O E$ e 4 non-carriers who did not perform moderate PA in midlife, APOE $e 4$ carriers who did not perform moderate PA in midlife had a 2.07-fold increased HR for MCI (95\%CI: 1.32, 3.26, $p<0.05)$, while APOE e4 carriers who performed moderate PA in midlife had a 1.53-fold increased HR for MCI (95\%CI: 1.10, 2.15, $p<0.05)$. When compared to $A P O E$ e 4 non-carriers who did not perform moderate PA in late life, $A P O E$ $e 4$ carriers who did not perform moderate PA in late life had a 1.89-fold increased $\mathrm{HR}$ for MCI (95\%CI: 1.37, 2.61, $p<0.05)$, and those APOE e4 carriers who performed moderate PA in late life had a 1.43-fold increased HR for MCI (95\%CI: 1.05, 2.95, $p<0.05)$ [48]. Woodard et al. revealed that the predicted probability of $\mathrm{CD}$ for APOE e4 carriers who reported low levels of PA ( $\leq 2$ days/week of low intensity) was significantly higher $(p=0.006)$ compared to APOE e4 non-carriers who reported low or high levels of PA ( $\leq 2$ days/week of low intensity, or $\geq 3$ days/week of moderate to heavy intensity, respectively) [51]. The article by Woodard et al. also shows that the predicted probability of CD for APOE e4 carriers who reported high levels of PA was not statistically different from that for APOE e4 non-carriers who reported low or high levels of PA $(p>0.05)$ [51]. Espeland et al. found that APOE $e 4$ carriers who were involved in the PA intervention group had reduced odds for $C D$ $(\mathrm{OR}=0.84,95 \% \mathrm{CI}: 0.52,1.36, p>0.05)$ as compared with non-carriers belonging to the control group [52].

Referring to the results provided in Table 2 that compare the risk of $\mathrm{CD}$ among APOE $e 4$ carriers who showed different PA levels, Woodard et al. revealed that the predicted probability of $\mathrm{CD}$ for $A P O E$ e 4 carriers who reported low levels of PA ( $\leq 2$ days/week of low intensity) was significantly higher $(p=0.006)$ compared to carriers who reported high levels of PA ( $\geq 3$ days/week of moderate to heavy intensity) [51]. Krell-Roesch et al. stratified their results based on the intensity of the PA (light, moderate and vigorous) and also based on the moment in life when PA was performed (never, only in midlife, only in late life, or always in midlife and late life). Based on this stratification, no significant 
differences for suffering MCI were found among carriers who performed or who did not perform light or moderate PA in different moments of their lifespan; however, those carriers who had performed vigorous intensity activity in midlife and late life (always) had lower risk of developing $\mathrm{MCI}$ in comparison with those who never performed vigorous PA: $\mathrm{HR}=0.46$ (95\%CI: $0.22,0.95, p<0.05)$ [48]. Niti et al. reported that participants who performed at least one PA had 0.34 odds for CD (95\%CI: 0.17, $0.68, p<0.05)$ compared with participants who did not perform any PA [49].

\section{Discussion}

In relation to the aims of this systematic review, the results support the notion that PA is a protective factor against $\mathrm{CD}$ in $A P O E$ e 4 carriers independently of the methodology used to assess the PA or the criteria to establish $C D$ and PA levels, since this is confirmed by four of the five studies included in this systematic review. It is remarkable in this first review of previous reports in the literature that PA was effective in preventing $C D$ in high-risk individuals such as APOE e4 carriers. However, there are several issues related to the methodology of included studies that must be taken into account in the interpretation of the results.

As can be seen in Tables 1 and 2, all the studies except one [51] showed results adjusted by different confounders, the most common being age and educational level. However, only two studies carried out a follow-up period $>5$ years [50,52]. Moreover, the short period of follow-up in some cases, the $>20 \%$ loss of participants, the self-reported PA and the fact that the results were not adjusted by important confounders such as age, educational level or cardiovascular risk factors could affect the results.

The methodology used to assess PA in the studies had some weaknesses, as the researchers used questionnaires, which are based on self-reported PA and, therefore, may over- or underestimate participants' PA. Some advantages of this type of questionnaire in studies such as those included in the present review are apparent, such as their simplicity, low cost, and ease of administration in large samples in a short period of time [54]. There is wide recognition that the choice of method may be a trade-off between accuracy level and feasibility [55], but when the aim is establishing a dose-response relationship, the use of motion sensors such as accelerometers would be important, although their use in large population studies is less feasible and they are not $100 \%$ accurate [54,56,57].

In addition, as can be seen in Tables 1 and 2, the use of different questionnaires to assess PA among studies, and the use of different cutoff points to define the different PA levels, make it difficult to compare among studies. Moreover, the heterogeneity in the method to assess cognitive function and in the definition and criteria used for establishing CD or $\mathrm{MCI}$ also complicates the comparison among studies. Nevertheless, the comparability of studies is supported to some extent because these criteria are commonly used in clinical practice to confirm the presence of $C D$.

Furthermore, most of the included studies only specified if the participants were $A P O E$ e 4 carriers, and therefore cases, or were APOE e4 non-carriers, and therefore controls, but did not specify whether there were any $A P O E e 2$ carriers in control groups. This could be of interest, because, as evidence suggests, $A P O E e 2$ has been associated with a reduced CD risk [58].

Despite these circumstances, all the included studies except one [52] support the idea that PA is a beneficial factor in terms of CD. The absence of significant differences in this study might be due to different reasons: first, the different type of sample used compared to the rest of the included studies, since all the participants had type 2 diabetes and overweight or obesity, and these are independent risk factors for CD [59] and can be improved with PA. Second, the intervention in this study may not be long enough to observe differences. Third, the results were only adjusted by age. 


\subsection{Physical Activity Dose and Risk of $C D$}

Regarding the results of the studies, this review found that some studies suggest that an increased amount and/or intensity of PA is more effective in reducing the risk of $\mathrm{CD}$ for APOE e4 carriers $[48,50,51]$. This finding may be explained with previous reports, which suggest that higher levels of PA may be associated with mitigating the increased risk of beta-amyloid deposition in APOE e4 carriers [60].

However, the sample size in studies such as the one by Woodard et al. was small [51], and PA amount and intensity were only self-reported, such that the precision of the doseresponse relationships may be affected. Therefore, although this review finds some support for the hypothesis that an increased amount and intensity of PA is more protective against $\mathrm{CD}$ in $A P O E$ e4 carriers, the evidence is limited, and new research may be needed to document the precise amount and intensity of PA to recommend in APOE e4 carriers.

It could be conceivable that previous studies in the general population, non-stratified by APOE e4 status, might shed some light on this subject. According to several longitudinal studies, exercise intensity might be more beneficial than duration regarding cognitive function in the general population [41,42]. However, analyzing some studies that reported results related to the amount and intensity of $\mathrm{PA}$ adequate for the prevention of $\mathrm{CD}$ in the general population, contradictory results were found. While some studies suggest that moderate intensity seems to be sufficient to show a beneficial effect, although higher intensity is more effective $[27,61]$, others stated that light intensity, such as a leisurely walk after dinner, is better than vigorous PA for the prevention of MCI [48].

It could be thought that the optimal dose of PA for $A P O E$ e4 persons to prevent $C D$ should not be very different from that of $A P O E$ e4 non-carriers. However, according to Shih et al., the same PA level in APOE e4 carriers and non-carriers results in a different risk reduction in $\mathrm{CD}$, obtaining greater benefits for non-carriers [50]. Therefore, it is possible that carriers should perform more PA to reduce the risk to the same extent as non-carriers, but, on the other hand, a study conducted by Schuit et al. reported that, when performing the same PA level in both groups, APOE e4 carriers obtain greater risk reduction for $\mathrm{CD}$ than non-carriers [62]. It is, therefore, apparent that new studies are required to determine the appropriate dose of PA to recommend in preventing CD.

\subsection{Strengths and Limitations}

The major strength of this review, which used the PRISMA system, is that, to our knowledge, it is the first systematic review focused on studies that stratify by apolipoprotein E genotype in relation to the association of PA and CD. Despite this, there are other limitations that must be considered. First, there is a heterogeneous methodology among the studies to assess the main variables and define the outcome. In the five included studies, a total of six different questionnaires were used to assess the PA, and a total of five different criteria were used to classify the participants in terms of PA levels; further, different criteria to diagnose $\mathrm{CD}$ were used in the included studies. Second, as discussed above, there are no studies that assess the PA with an objective method. Third, there are few studies in the literature related to our topic that show results by subgroups based on PA levels and the $A P O E$ e4 status of the participants, and studies such as the one by Woodard et al. were carried out on small samples.

\subsection{Future Recommendations}

Although there is some evidence indicating that PA can be a protective factor against $\mathrm{CD}$ in APOE e4 persons, future research will be needed in order to corroborate this. It seems that the literature is mainly limited by the failure to present data separately for $A P O E$ e 4 carriers and non-carriers, and more studies that stratify in groups according to the genotype and PA levels of the participants should, therefore, be conducted. It is also important to perform studies that evaluate PA objectively by means of motion sensors such as accelerometry, as, despite some limitations, it allows a more precise assessment of the amount and intensity of PA. It would also be interesting to perform intervention studies, 
ideally RCTs, in APOE e4 persons in order to provide data on the amount and intensity of PA that will be optimal for the prevention/slowdown of CD in this type of population. Currently, some clinical trials such as U.S. POINTER, IGNITE and PAAD-2 [63-65] are focused on the role that PA and other lifestyle variables have in cognition among different types of population, including APOE e4 carriers. The results of these clinical trials could shed some light on this area.

\section{Conclusions}

The results of the studies included in this systematic review support the idea that $\mathrm{PA}$ is a protective factor against $\mathrm{CD}$ in individuals of high genetic risk, specifically $A P O E$ $e 4$ carriers. These findings have high clinical and public health significance. Moreover, the results suggest that in this population, a higher dose of PA (amount and/or intensity) might have greater benefits, but it would be necessary to carry out further studies that would allow these findings to be contrasted, since the existing evidence is limited. Further studies should try to establish the optimal dose of PA to effectively and efficiently prevent $\mathrm{CD}$ in $A P O E$ e 4 carriers.

Supplementary Materials: The following are available online at https:/ /www.mdpi.com/article/10.339 0/ijerph18147238/s1, Text S1: Quality assessment information. Newcastle-Ottawa Scale (NOS) criteria.

Author Contributions: Conceptualization, J.L.P.-L., J.A.C. (Jose Antonio Casajús), J.A.C. (José Antonio Casasnovas), A.L., E.L., B.M.-F. and A.G.-A.; methodology, J.L.P.-L., J.A.C. (Jose Antonio Casajús), B.M.-F. and A.G.-A.; formal analysis, J.L.P.-L., J.A.C. (Jose Antonio Casajús) and A.G.-A.; investigation, J.L.P.-L., J.A.C. (Jose Antonio Casajús), J.M.A.-M., B.M.-F. and A.G.-A.; writing-original draft preparation, J.L.P.-L. and A.G.-A.; writing-review and editing, J.A.C. (Jose Antonio Casajús), J.A.C. (José Antonio Casasnovas), J.M.A.-M., A.L., E.L. and B.M.-F.; visualization, J.L.P.-L. and A.G.-A.; supervision, J.L.P.-L. All authors have read and agreed to the published version of the manuscript.

Funding: This study was supported in part by CIBERCV and grant PI19/00948 from the Instituto de Salud Carlos III (co-supported by the European Regional Development Fund "Investing in your future"). J.L.P.-L. received a Grant FPU 2016 (FPU16/02539) from the Ministerio de Educación Cultura y Deporte (Spain).

Institutional Review Board Statement: Not applicable.

Informed Consent Statement: Not applicable.

Data Availability Statement: Not applicable.

Conflicts of Interest: The authors declare no conflict of interest.

\section{References}

1. Li, J.; Han, X.; Zhang, X.; Wang, S. Spatiotemporal evolution of global population ageing from 1960 to 2017. BMC Public Health 2019, 19, 1-15. [CrossRef]

2. Lipnicki, D.M.; Makkar, S.R.; Crawford, J.D.; Thalamuthu, A.; Kochan, N.A.; Lima-costa, M.F.; Castro-costa, E.; Pinheireo Ferri, C.; Brayne, C.; Stephan, B.; et al. Determinants of cognitive performance and decline in 20 diverse ethno-regional groups: A COSMIC collaboration cohort study. PLoS Med. 2019, 16, 1-27. [CrossRef]

3. Murman, D.L. The Impact of Age on Cognition. Semin. Hear. 2015, 36, 111-121. [CrossRef] [PubMed]

4. Deep, C.A.; Jeste, D.V. Definitions and Predictors of Successful Aging: A Comprehensive Review of Larger Quantitative Studies. Am. J. Geriatr. Psychiatry 2006, 14, 6-20. [CrossRef]

5. WHO. Decade of Healthy Ageing 2020-2030; World Health Organization: Madrid, Spain, 2020.

6. Bousquet, J.; Malva, J.; Nogues, M.; Rodriguez, L.; Vellas, B.; Farrell, J. Operational Definition of Active and Healthy Aging (AHA): The European Innovation Partnership (EIP) on AHA Reference Site Questionnaire: Montpellier 20-21 October 2014, Lisbon 2 July 2015. J. Am. Med. Dir. Assoc. 2015, 16, 1020-1026. [CrossRef] [PubMed]

7. Geslani, D.M.; Tierney, C.; Szalai, J.P. Mild Cognitive Impairment: An Operational Defi nition and Its Conversion Rate to Alzheimer' s Disease. Dement. Geriatr. Cogn. Disord. 2005, 19, 383-389. [CrossRef] [PubMed]

8. Yates, J.A.; Clare, L.; Woods, R.T. What is the Relationship between Health, Mood, and Mild Cognitive Impairment? J. Alzheimer's Dis. 2017, 55, 1183-1193. [CrossRef] 
9. Santabárbara, J.; Lopez-Anton, R.; Marcos, G.; De-la-Camara, C.; Lobo, E.; Saz, P.; Gracia-García, P.; Ventura, T.; Campayo, A.; Rodríguez-Mañas, L.; et al. Degree of cognitive impairment and mortality: A 17-year follow-up in a community study. Epidemiol. Psychiatr. Sci. 2015, 24, 503-511. [CrossRef] [PubMed]

10. Santabárbara, J.; Garcia-García, P.; Pírez, G.; López-antón, R.; Concepcion De La Cámara, M.; Ventura, T.; Pérez-Sastre, M.; Lobo, E.; Saz, P.; Marcos, G.; et al. Mortality in Mild Cognitive Impairment Diagnosed with DSM-5 Criteria and with Petersen' s Criteria: A 17-Year Follow-Up in a Community Study. Am. J. Geriatr. Psychiatry 2016, 24, 977-986. [CrossRef] [PubMed]

11. Gracia-García, P.; López-antón, R.; Santabárbara, J.; Quintanilla, M.A.; De-la-Cámara, C.; Marcos, G.; Lobo, E.; Lobo, A.; The ZARADEMP Workgroup. Cognition and daily activities in a general population sample aged +55 . Aging Neuropsychol. Cogn. 2020, 4, 1-14. [CrossRef]

12. Etgen, T.; Sander, D.; Bickel, H.; Förstl, H. Mild Cognitive Impairment and Dementia: The importance of modifiable risk factors. Dtsch. Aerzteblatt. Int. 2011, 108, 743-750.

13. Czyz-Szypenbejl, K.; Medrzycka-Dabrowska, W.; Kwiecien-Jagus, K.; Lewandowska, K. The ocurrence of postoperative cognitive dysfunction (POCD)—Systematic Review. Psychiatr. Pol. 2019, 53, 145-160. [CrossRef]

14. Baumgart, M.; Snyder, H.M.; Carrillo, M.C.; Fazio, S.; Kim, H.; Johns, H. Summary of the evidence on modifiable risk factors for cognitive decline and dementia: A population-based perspective. Alzheimer's Dement. 2015, 11, 718-726. [CrossRef]

15. Gao, Y.; Huang, C.; Zhao, K.; Ma, L.; Qiu, X.; Zhang, L.; Xiu, Y.; Chen, L.; Lu, W.; Huang, C.; et al. Depression as a risk factor for dementia and mild cognitive impairment: A meta-analysis of longitudinal studies. Int J. Geriatr. Psychiatry 2013, 28, 441-449. [CrossRef] [PubMed]

16. Gulpers, B.; Ramakers, I.; Hamel, R.; Köhler, S.; Oude Voshaar, R.; Verhey, F. Anxiety as a Predictor for Cognitive Decline and Dementia: A Systematic Review and Meta-Analysis. Am. J. Geriatr. Psychiatry 2016, 24, 823-842. [CrossRef] [PubMed]

17. Rawle, M.J.; Davis, D.; Bendayan, R.; Wong, A. Apolipoprotein-E (Apoe) $\varepsilon 4$ and cognitive decline over the adult life course. Transl. Psychiatry 2018, 8, 1-8. [CrossRef]

18. Kern, S.; Mehlig, K.; Kern, J.; Zetterberg, H.; Thelle, D.; Skoog, I.; Lissner, L.; Blennow, K.; Börjesson-Hanson, A. The distribution of alipoprotein E genotype over the adult lifespan and in relation to country of birth. Am. J. Epidemiol. 2015, 181, $214-217$. [CrossRef] [PubMed]

19. Khan, T.A.; Shah, T.; Prieto, D.; Zhang, W.; Price, J.; Fowkes, G.R.; Cooper, J.; Talmud, P.J.; Humphires, S.E.; Sundstrom, J.; et al. Apolipoprotein E genotype, cardiovascular biomarkers and risk of stroke: Systematic review and meta-analysis of 14,015 stroke cases and pooled analysis of primary biomarker data from up to 60,883 individuals. Int. J. Epidemiol. 2013, 42, 475-492. [CrossRef] [PubMed]

20. Mahley, R.W. Apolipoprotein E: From cardiovascular disease to neurodegenerative disorders. J. Mol. Med. 2016, 94, 739-746. [CrossRef]

21. Neu, S.C.; Pa, J.; Kukull, W.; Beekly, D.; Kuzma, A.; Gangadharan, P.; Wang, L.S.; Romero, K.; Arneric, S.P.; Redolfi, A.; et al. Apolipoprotein E genotype and sex risk factors for Alzheimer disease: A meta-analysis. JAMA Neurol. 2017, 74, 1178-1189. [CrossRef]

22. Shi, J.; Liu, Y.; Liu, Y.; Li, Y.; Qiu, S.; Bai, Y.; Gu, Y.; Luo, J.; Cui, H.; Li, Y.; et al. Association between ApoE polymorphism and Hypertension: A Meta-analysis of 28 studies including 5898 cases and 7518 controls. Gene 2018, 30, 197-207. [CrossRef]

23. Hayden, K.M.; Lutz, M.W.; Kuchibhatla, M. Effect of APOE and CD33 on Cognitive Decline. PLoS ONE 2015, 10, 1-10. [CrossRef]

24. Makkar, S.R.; Lipnicki, D.M.; Crawford, J.D.; Kochan, N.A.; Castro-costa, E.; Fernandez Lima-Costa, M.; Diniz, B.S.; Brayne, C.; Stephan, B.; Matthews, F.; et al. APOE e4 and the influence of sex, age, vascular risk factors, and ethnicity on cognitive decline. J. Gerontol. Ser. A 2020, 75, 1863-1873. [CrossRef] [PubMed]

25. Chia-Chen, L.; Takahisa, K.; Huaxi, X.; Guojun, B. Apolipoprotein E and Alzheimer disease: Risk, mechanisms, and therapy. Nat. Rev. Neurol. 2013, 9, 106-118.

26. Gardener, H.; Wright, C.B.; Dong, C.; Cheung, K.; Derosa, J.; Nannery, M.; Stern, Y.; Elkind, M.S.V.; Sacco, R.L. Ideal Cardiovascular Health and Cognitive Aging in the Northern Manhattan Study. J. Am. Heart Assoc. 2016, 16, 1-11. [CrossRef] [PubMed]

27. Blondell, S.J.; Hammersley-Mather, R.; Veerman, J.L. Does physical activity prevent cognitive decline and dementia? A systematic review and meta-analysis of longitudinal studies. BMC Public Health 2014, 14, 1-12. [CrossRef] [PubMed]

28. Lindenberger, U. Human cognitive aging: Corriger la fortune? Science 2014, 346, 572-578. [CrossRef] [PubMed]

29. Kennedy, G.; Hardman, R.J.; MacPherson, H.; Scholey, A.B.; Pipingas, A. How Does Exercise Reduce the Rate of Age-Associated Cognitive Decline? A Review of Potential Mechanisms. J. Alzheimer's Dis. 2017, 55, 1-18. [CrossRef]

30. Macpherson, H.; Teo, W.-P.; Schneider, L.A.; Smith, A.E. A Life-Long Approach to Physical Activity for Brain Health. Front. Aging Neurosci. 2017, 9, 1-12. [CrossRef]

31. McKee, A.C.; Daneshvar, D.H.; Alvarez, V.E.; Stein, T.D. The neuropathology of sport. Acta Neuropathol. 2014, $127,29-51$. [CrossRef]

32. Elias, M.F.; Sullivan, L.M.; D'Agostino, R.B.; Elias, P.K.; Beiser, A.; Au, R.; Seshadri, S.; DeCarli, C.; Wolf, P.A. Framingham Stroke Risk Profile and Lowered Cognitive Performance. Stroke 2004, 35, 404-409. [CrossRef]

33. Jefferson, A.L.; Hohman, T.J.; Liu, D.; Haj-Hassan, S.; Gifford, K.A.; Benson, E.M.; Skinner, J.S.; Lu, Z.; Sparling, J.; Sumner, E.C.; et al. Adverse vascular risk is related to cognitive decline in older adults. J. Alzheimer's Dis. 2015, 44, 1361-1373. [CrossRef] 
34. Kraus, W.E.; Powell, K.E.; Haskell, W.L.; Janz, K.F.; Wayne, W.; Jakicic, J.M.; Troiano, R.P.; Sprow, K.; Torres, A.; Piercy, K.L.; et al. Physical activity, all-cause and cardiovascular mortality, and cardiovascular disease. Med. Sci. Sports Exerc. 2020, 51, 1270-1281. [CrossRef] [PubMed]

35. Pedersen, B.K.; Saltin, B. Exercise as medicine-Evidence for prescribing exercise as therapy in 26 different chronic diseases. Scand. J. Med. Sci. Sports 2015, 25, 1-72.

36. Wahid, A.; Manek, N.; Nichols, M.; Kelly, P.; Foster, C.; Webster, P.; Kaur, A.; Friedemann Smith, C.; Wilkins, E.; Rayner, M.; et al Quantifying the Association Between Physical Activity and Cardiovascular Disease and Diabetes: A Systematic Review and Meta-Analysis. J. Am. Heart Assoc. 2016, 5, 1-32. [CrossRef]

37. Rebar, A.L.; Stanton, R.; Geard, D.; Short, C.; Duncan, M.J.; Vandelanotte, C. A meta-meta-analysis of the effect of physical activity on depression and anxiety in non-clinical adult populations. Health Psychol. Rev. 2015, 9, 366-378. [CrossRef] [PubMed]

38. Gomes-Osman, J.; Cabral, D.F.; Morris, T.P.; McInerney, K.; Cahalin, L.P.; Rundek, T.; Oliveira, A.; Pascual-Leone, A. Exercise for cognitive brain health in aging: A systematic review for an evolution of dose. Neurol. Clin. Pract. 2018, 8, 1-9. [CrossRef]

39. Li, Z.; Peng, X.; Xiang, W.; Han, J.; Li, K. The effect of resistance training on cognitive function in the older adults: A systematic review of randomized clinical trials. Aging Clin. Exp. Res. 2018, 13, 1-15. [CrossRef] [PubMed]

40. Sofi, F.; Valecchi, D.; Bacci, D.; Abbate, R.; Gensini, G.F.; Casini, A.; Macchi, C. Physical activity and risk of cognitive decline: A meta-analysis of prospective studies. J. Intern. Med. 2011, 269, 107-117. [CrossRef]

41. Angevaren, M.; Vanhees, L.; Wendel-vos, W.; Verhaar, H.J.J.; Aufdemkampe, G.; Aleman, A.; Verschuren, W.M.M. Intensity, but not duration, of physical activities is related to cognitive function. Eur. J. Cardiovasc. Prev. Rehabil. 2007, 14, 825-830. [CrossRef]

42. van Gelder, B.M.; Tijhuis, M.A.R.; Kalmijn, S.; Giampaoli, S.; Nissinen, A.; Kromhout, D. Physical activity in relation to cognitive decline in elderly men: The FINE Study. Neurology 2004, 63, 2316-2321. [CrossRef] [PubMed]

43. Flicker, L.; Almeida, O.P.; Acres, J.; Le, M.T.; Tuohy, R.J.; Jamrozik, K.; Hankey, G.; Norman, P. Predictors of impaired cognitive function in men over the age of 80 years: Results. Age Ageing 2005, 34, 77-80. [CrossRef] [PubMed]

44. Laurin, D.; Verreault, R.; Lindsay, J.; MacPherson, K.; Rockwood, K. Physical Activity and Risk of Cognitive Impairment and Dementia in Elderly Persons. Arch. Neurol. 2001, 58, 498-504. [CrossRef] [PubMed]

45. Moher, D.; Liberati, A.; Tetzlaff, J.; Altman, D.G.; The PRISMA Group. Preferred reporting items for systematic reviews and meta-analyses: The PRISMA statement. PLoS Med. 2009, 6, e1000097. [CrossRef]

46. Wells, G.; Shea, B.; O'Connell, D.; Peterson, J.; Welch, V.; Losos, M.; Tugwell, P. The Newcastle-Ottawa Scale (NOS) for Assessing the Quality of Nonrandomised Studies in Meta-Analyses. Available online: http://www.ohri.ca/programs/clinical_ epidemiology / oxford.asp (accessed on 10 October 2020).

47. de Morton, N. The PEDro scale is a valid measure of the methodological quality of clinical trials: A demographic study. Aust. J. Physiother. 2009, 55, 129-133. [CrossRef]

48. Krell-Roesch, J.; Pink, A.; Roberts, R.O.; Stokin, G.B.; Mielke, M.M.; Spangehl, K.A.; Bartley, M.M.; Knopman, D.S.; Christianson, T.J.H.; Petersen, R.C.; et al. Timing of Physical Activity, Apolipoprotein E $\varepsilon 4$ Genotype, and Risk of Incident Mild Cognitive Impairment. J. Am. Geriatr. Soc. 2016, 64, 2479-2486. [CrossRef] [PubMed]

49. Niti, M.; Yap, K.B.; Kua, E.H.; Tan, C.H.; Ng, T.P. Physical, social and productive leisure activities, cognitive decline and interaction with APOE- $\varepsilon 4$ genotype in Chinese older adults. Int. Psychogeriatr. 2008, 20, 237-251. [CrossRef]

50. Shih, I.F.; Paul, K.; Haan, M.; Yu, Y.; Ritz, B. Physical activity modifies the influence of apolipoprotein E $\varepsilon 4$ allele and type 2 diabetes on dementia and cognitive impairment among older Mexican Americans. Alzheimer's Dement. 2018, 14, 1-9. [CrossRef]

51. Woodard, J.; Nielson, K.; Sugarman, M.; Smith, C.; Seidenberg, M.; Durgerian, S.; Butts, A.; Hantke, N.; Lancaster, M.; Matthews, M.A.; et al. Lifestyle and genetic contributions to cognitive decline and hippocampal integrity in healthy aging. Curr. Alzheimer Res. 2012, 9, 436-446. [CrossRef]

52. Espeland, M.A.; Luchsinger, J.A.; Baker, L.D.; Neiberg, R.; Kahn, S.E.; Arnold, S.E.; Wing, R.R.; Blackburn, G.L.; Bray, G.; Evans, M.; et al. Effect of a long-term intensive lifestyle intervention on prevalence of cognitive impairment. Neurology 2017, 88, 2026-2035. [CrossRef]

53. Ciesielska, N.; Sokołowski, R.; Mazur, E.; Podhorecka, M.; Polak-Szabela, A.; Kędziora-Kornatowska, K. Is the Montreal Cognitive Assessment (MoCA) test better suited than the Mini-Mental State Examination (MMSE) in mild cognitive impairment (MCI) detection among people aged over 60? Meta-analysis. Psychiatr Pol. 2016, 50, 1039-1052. [CrossRef] [PubMed]

54. Ainsworth, B.; Cahalin, L.; Buman, M.; Ross, R. The Current State of Physical Activity Assessment Tools. Prog. Cardiovasc. Dis. 2015, 57, 387-395. [CrossRef]

55. Warren, J.M.; Ekelund, U.; Besson, H.; Mezzani, A.; Geladas, N.; Vanhees, L. Assessment of physical activity-A review of methodologies with reference to epidemiological research: A report of the exercise physiology section of the European Association of Cardiovascular Prevention and Rehabilitation. Eur. J. Cardiovasc. Prev. Rehabil. 2010, 17, 127-139. [CrossRef] [PubMed]

56. Skender, S.; Ose, J.; Chang-claude, J.; Paskow, M.; Brühmann, B.; Siegel, E.M.; Steindorf, K.; Ulrich, C.M. Accelerometry and physical activity questionnaires-A systematic review. BMC Public Health 2016, 16, 1-10. [CrossRef] [PubMed]

57. Welk, G.J. Harmonizing Monitor- and Report-Based Estimates of Physical Activity through Calibration. Kinesiol. Rev. 2019, 8, 1-9. [CrossRef]

58. Suri, S.; Heise, V.; Trachtenberg, A.J.; Mackay, C.E. The forgotten APOE allele: A review of the evidence and suggested mechanisms for the protective effect of APOE e2. Neurosci. Biobehav. Rev. 2013, 37, 2878-2886. [CrossRef] 
59. Bangen, K.; Gu, Y.; Gross, A.; Schneider, B.; Skinner, J.; Benitez, A.; Sachs, B.C.; Shih, R.; Sisco, S.; Schupf, N.; et al. Relation of Type 2 Diabetes with Cognitive Change in a Multiethnic Elderly Cohort HHS Public Access. J. Am. Geriatr. Soc. 2015, 63, 1075-1083. [CrossRef] [PubMed]

60. Brown, B.M.; Peiffer, J.J.; Taddei, K.; Lui, J.K.; Laws, S.M.; Gupta, V.B.; Taddei, T.; Ward, V.K.; Rodrigues, M.A.; Burnham, S.; et al Physical activity and amyloid- $\mathrm{b}$ plasma and brain levels: Results from the Australian Imaging, Biomarkers and Lifestyle Study of Ageing. Mol. Psychiatry 2012, 18, 875-881. [CrossRef]

61. Guure, C.B.; Ibrahim, N.A.; Adam, M.B.; Said, S.M. Impact of Physical Activity on Cognitive Decline, Dementia, and Its Subtypes: Meta-Analysis of Prospective Studies. Biomed. Res. Int. 2017, 2017, 1-13. [CrossRef] [PubMed]

62. Schuit, A.J.; Feskens, E.J.M.; Launer, L.J.; Kromhout, D. Physical activity and cognitive decline, the role of the apolipoprotein e4 allele. Med. Sci. Sports Exerc. 2001, 33, 772-777. [CrossRef]

63. U.S. POINTER Alzheimer's Association. Available online: https://uspointer.net/about.cfm (accessed on 17 November 2020).

64. Erickson, K.I.; Grove, G.A.; Burns, J.M.; Hillman, C.H.; Kramer, A.F.; McAuley, E.; Vidoni, E.D.; Becker, J.T.; Butters, M.A.; Gray, K.; et al. Investigating Gains in Neurocognition in an Intervention Trial of Exercise (IGNITE): Protocol. Contemp. Clin. Trials. 2019, 85, 1-11. [CrossRef] [PubMed]

65. Park, K.S.; Ganesh, A.B.; Berry, N.T.; Mobley, Y.P.; Karper, W.B.; Labban, J.D.; Wahlheim, C.N.; Williams, T.M.; Wideman, L.; Etnier, J.L. The effect of physical activity on cognition relative to APOE genotype (PAAD-2): Study protocol for a phase II randomized control trial. BMC Neurol. 2020, 20, 1-15. [CrossRef] [PubMed] 